\title{
Circulating MicroRNA Profiles Differ between Qi-Stagnation and Qi-Deficiency in Coronary Heart Disease Patients with Blood Stasis Syndrome
}

\author{
Jincai Hou, ${ }^{1,2}$ Jun Wang, ${ }^{3}$ Chengren Lin, ${ }^{1,2}$ Jianhua Fu, ${ }^{1,2}$ Jianxun Ren, ${ }^{1,2}$ Lei Li, ${ }^{1,2}$ \\ Hao Guo, ${ }^{1,2}$ Xiao Han, ${ }^{1,2}$ and Jianxun Liu ${ }^{1,2}$ \\ ${ }^{1}$ Xiyuan Hospital of China Academy of Chinese Medical Sciences, 1 Xiyuan Caochang, Haidian District, Beijing 100091, China \\ ${ }^{2}$ Beijing Key Laboratory of Pharmacology of Chinese Materia Medica, Institute of Basic Medical Sciences of Xiyuan Hospital, \\ 1 Xiyuan Caochang, Haidian District, Beijing 100091, China \\ ${ }^{3}$ Institute of Basic Theory, China Academy of Chinese Medical Sciences, 16 Dong Zhi Men Nei Nan Xiao Jie, Beijing 100700, China
}

Correspondence should be addressed to Jianxun Liu; ljxunljxun@163.com

Received 14 August 2014; Accepted 6 November 2014; Published 4 December 2014

Academic Editor: Karl Wah-Keung Tsim

Copyright (c) 2014 Jincai Hou et al. This is an open access article distributed under the Creative Commons Attribution License, which permits unrestricted use, distribution, and reproduction in any medium, provided the original work is properly cited.

\begin{abstract}
We compared the circulating microRNA profiles of Qi-stagnation (QSB) and Qi-deficiency (QDB) in coronary heart disease (CHD) patients with blood stasis syndrome. Twenty-nine CHD patients were divided into QSB group and QDB group. The analysis was carried out through comparing their circulating microRNA profiles and the following bioinformatics analysis. The number of differential miRNAs in QDB group was much more than that in QSB group. Functional annotations of the differentially expressed miRNAs target genes in the QSB group and QDB group were, respectively, related to regulation of cellular component organization, regulation of glucose metabolic process, and so forth and protein kinase cascade, phosphate metabolic process, and so forth. KEGG pathway analysis showed that the process Qi-deficiency was associated with phagocytosis including endocytosis and mTOR signaling pathway. Specifically, pathway of cell adhesion molecules played the crucial role in the pathological process of Qistagnation, with a unique upregulation except for pathways associated with cancer signal. MicroRNA-gene-net analysis indicated that let-7c, miR-4487, miR-619, miR-8075, miR-6735, and miR-32-5p and miR-17-5p, miR-130a, and miR 320 family had the most important and extensive regulatory function for Qi-stagnation syndromes and Qi-deficiency syndromes, respectively. Differentially expressed miRNAs and concerned pathways suggest different molecular mechanisms that may mediate the pathological process of QSB and QDB syndromes.
\end{abstract}

\section{Introduction}

Coronary heart disease (CHD), characterized by myocardial ischemia or necrosis caused by vascular stenosis or occlusion, is one of the leading causes of deaths and hospital admissions worldwide and constitutes the leading cause of disease burden in the world according to the 2010 Global Burden of Disease Study (GBD) $[1,2]$. Traditional Chinese medicine (TCM), with a 3000-year-old history that includes unique theories for aetiology and systems of diagnosis and treatment has been proven to be an effective classification method in patient stratification integrated with biomedical diagnostic method $[3,4]$. TCM patterns have been used in China for thousands of years and are still playing an important role in the treatment of chronic diseases such as CHD. In TCM, the diagnosis, clinical evaluation, and treatment of $\mathrm{CHD}$ are based on signs and subjective symptoms according to the unique concept of "wholism." CHD treatments are based on TCM diagnostics and syndrome differentiation which are the comprehensive responses of a certain stage in the disease process [5].

Chinese medicine holds that blood stasis syndrome is a common reason responsible for CHD in clinic of Chinese medicine due to Qi-stagnation (QSB) or Qi-deficiency (QDB). At present, many researchers consider that objective signs of blood stasis are reflected in microcirculation related 
to vessel and cell function, such as vascular diastolic dysfunction, abnormal platelet function, blood viscosity, and blood cell adhesion. As for QSB and QDB, there exists different biological basis and objective signs, with their respective unique characteristics of pathological changes.

MicroRNAs (miRNAs) are endogenous small RNA molecules best known for their function in posttranscriptional gene regulation. More than $60 \%$ of protein-coding genes may be targeted by miRNAs [6], mainly through translational repression and degradation of target mRNAs. MiRNAs are pivotal modulators of mammalian cardiovascular development and disease and can be steadily found in the systemic circulation of both animals and humans, where they show a remarkable stability probably due to internalization in vesicles and binding to circulating proteins and other molecules [7]. Since their levels may significantly change upon stress, circulating miRNAs have been proposed as diagnostic biomarkers in different pathologic conditions [8].

The characterization and differentiation of QSB and QDB syndromes have played an important role in the clinical practice of TCM for CHD. We speculated that miRNAs of peripheral blood have been a major parameter in discriminating the QSB and QDB syndromes and affected the appearance of the CHD patients with QSB and QDB syndromes. Therefore, we investigated the possible relationship between the syndromes as described in TCM practice and miRNAs profiles to bridge the gap between traditional syndrome diagnosis and molecular systems biology.

\section{Materials and Methods}

2.1. Participant Recruitment. This study was approved by the Medical Ethical Committee Xiyuan Hospital of China Academy of Chinese Medical Sciences (2011XL008-2). All 19 healthy volunteers reported no CHD and exhibited a normal syndrome as judged by TCM doctors. Delayed, written informed consent was obtained from all enrollees after they were clinically stabilized. 29 patients presenting with CHD were identified in Xiyuan Hospital of China Academy of Chinese Medical Sciences during the time between March 2012 and June 2013. Of the 29 patients screened for inclusion, 7 fulfilled study inclusion criteria for QSB group and 22 fulfilled study inclusion criteria for QDB group.

2.2. Sample Acquisition and Handling. 10 milliliters of venous blood was obtained from ulnar vein and transferred in a sterile fashion into vacuum blood collection tubes. Samples were placed immediately on ice and then centrifuged at $4^{\circ} \mathrm{C}$ for $20 \mathrm{~min}$ at $3000 \mathrm{rpm}$ to obtain the serum which was taken to the study laboratory within 10 minutes.

\subsection{RNA Isolation and RNA Amplification. Total RNA} including small RNA and miRNAs was isolated from serum samples by TRIzol reagent (Invitrogen, Canada) and purified using RNeasy Mini Kit (Qiagen, German), including a DNase digestion treatment. We ensure that the purification method retains slow molecular weight (LMW) RNA. RNA concentrations were determined by the absorbance at $260 \mathrm{~nm}$ and quality control standards were $A_{260} / A_{280}=1.8-2.1$, using NanoDrop 2000 (Thermo, America).

2.4. Affymetrix miRNA Microarray. RNA was labeled using FlashTag Biotin HSR labeling kit as the manufacturer's instructions (Genisphere, America). Labeled RNA was hybridized to GeneChip microRNA 3.0 array (Affymetrix, America) according to the user manuals. Affymetrix Expression Console Software (version 1.3.1) was used for microarray analysis, including data normalization, summarization, and quality control assessment. Median-centric normalization was used for the custom microRNA oligonucleotide chips. Affymetrix chips were normalized using the robust multichip analysis (RMA) procedure. Differentially expressed miRNAs were identified based on RVM $t$-test analysis. Differentially expressed miRNAs with at least 1.5 -fold change in either direction with $P<0.05$ were considered to be up- or downregulated.

2.5. Quantitative Real Time RT-PCR Validation. The cDNA was subjected to real time quantitative PCR with defined primers and Power SYBR Green PCR Master Mix (Applied Biosystems, Foster City, CA, USA). The data were analyzed using the ABI 7000 system SDS software (Applied Biosystems, Foster City, CA, USA). All the experiments were performed in duplicate and relative expression levels of these microRNAs were determined by the $2^{-\Delta \Delta \mathrm{Ct}}$ method.

2.6. Gene Ontology (GO) Analysis. Gene ontology analysis has been carried out using DAVID and GSEA. ID of differential expressed genes was uploaded to the DAVID database and the analysis was performed based on their respective molecular function, biological process, or cellular component. Functional categories were enriched within genes that were differentially expressed between the QSB and QDB groups. Top ten GO functional categories that genes mainly involved in were selected for specific analysis.

2.7. Pathway Analysis. Normalised signal intensities from each experimental condition for the differentially expressed genes were uploaded to the KEGG database which is to collect pathway maps that computerize the network information of molecular interaction. KEGG analysis (KEGG data version: Release 70.1, June 1, 2014) is used for discovering the relation that is not easily visible from the changes of individual genes. Pathways that had significant changes of $P<0.05$ and fold change $>1.5$ were identified for further analysis.

2.8. MicroRNA-Gene-Net Analysis. To build a miRNA-genenetwork, the relationship between miRNAs and genes was counted by their differential expression values and according to their interactions in the Sanger (Targetscan\&miRanda) miRNA database. TargetScan 6.2 (http://www.targetscan.org/) in conjunction with the miRanda version August 2010 Release (available at: http://www.microrna.org/) was used to predict the targets of the miRNAs. The circles represented target genes and the squares represented miRNAs. The relationships between miRNAs and target genes 
TABLE 1: Demographic characteristics of CHD patients with QSB and QDB syndrome.

\begin{tabular}{lcc}
\hline & QSB $(N=7)$ & QDB $(N=22)$ \\
\hline Demographics: & & $65.43 \pm 9.5$ \\
Mean age (years) & 4 & $66.55 \pm 11.7$ \\
Sex (Male) & $83.2 \pm 6.8$ & $77.8 \pm 10.7$ \\
Heart rate (BPM) & $144 \pm 12.1 / 90.6 \pm 11.3$ & $140.5 \pm 5.23 / 81.8 \pm 4.1$ \\
Blood pressure (SBP/DBP) & $27.9 \pm 0.6$ & $26.2 \pm 0.85$ \\
Body mass index $\left(\mathrm{kg} / \mathrm{m}^{2}\right)$, mean \pm SE & & $15(68)$ \\
Comorbid conditions: & $3(42)$ & $4(18)$ \\
Hypertension & $1(14)$ & $4(18)$ \\
Diabetes mellitus & $1(14)$ & $13(59)$ \\
Hyperlipidemia & $4(57)$ & $6(27)$ \\
Current smoking & $2(28)$ & $20(90)$ \\
Alcohol & & $5(22)$ \\
Prehospital medications: & $5(71)$ & $8(36)$ \\
Statins & $1(14)$ & $6(27)$ \\
Beta blocker & $2(28)$ & $9(41)$ \\
Angiotensin-converting enzyme inhibitor/angiotensin receptor blocker & $2(28)$ & $7(32)$ \\
Calcium channel blocker & $3(43)$ & $3(43)$ \\
Aspirin & & \\
Nitroglycerin & &
\end{tabular}

were represented by edges. The center of the network was represented by degree which is the contribution of one miRNA to the genes around or the contribution of one gene to the miRNAs around [9]. The key miRNA and gene in the network always have the biggest degrees.

\section{Results}

3.1. Patient Demographic. As shown in Table 1, the age of QSB group $(65.43 \pm 9.55)$ had no significant difference compared with QDB $(66.55 \pm 11.74)$. Most participants presenting with QSB and QDB syndromes in our cohort were uniformly male, with the percentage of $71.43 \%$ in QSB group and $63.64 \%$ in QDB group. 57.1\% of QSB patients and 72.7\% of QDB patients had complications including hypertension, diabetes, and hyperlipidemia. The majority of participants in both QSB and QDB groups had a history of hypertension $(60 \%$ and $93.7 \%$, resp.) and a minority had a history of diabetes or hyperlipidemia ( $40 \%$ and $16.3 \%$, resp.).

\subsection{Identification of miRNAs from QSB and QDB Syndromes} by miRNA Array. MiRNAs that are differentially expressed between QSB and QDB groups which are expected to contribute to functional differences among the CHD patients. Therefore, we identified miRNAs that are differentially expressed $(P<0.05)$ between QSB and QDB by microarrays. Compared with the healthy patients, we detected 21 differentially expressed miRNAs, with 16 (76.2\%) upregulated miRNAs and 5 (23.8\%) downregulated miRNAs. 33 differentially expressed miRNAs were found in QDB group, with $21(63.7 \%)$ upregulated miRNAs and 12 (36.3\%) downregulated miRNAs. Figure 1 displayed the differentially expressed miRNAs overlapping and nonoverlapping in QSB and QDB groups, which showed that 5 upregulated (Figure 1(a)) and 4 downregulated (Figure 1(b)) differentially expressed miRNAs overlapping among the 2 groups.

3.3. $q R T-P C R$ Validation. To determine that the microarraybased miRNAs detections were reliable for the determination of miRNAs expression patterns in the serum, we performed SYBR green-based real time quantitative PCR on upregulated or downregulated miRNAs in the QSB and QDB groups from microarray data (FDR $P<0.05$; fold change $>1.5$ ). All randomly selected miRNAs were in agreement with the microarray analyses in terms of the direction of the observed differential expression (Figure 1(c)). Specifically, the observed $\log _{2}$ fold changes with qRT-PCR of the upregulated miRNAs (miR-45la, miR-23b, miR-455, and miR-32) were the same positive tendency as obtained with the microarray data. Equally, the observed $\log _{2}$ fold changes with qRT-PCR of the down-regulated miRNAs (miR-320c and miR-619) were the same negative tendency as obtained with the microarray data.

3.4. Gene Ontology (GO) Analysis of the miRNAs Target Genes. In an effort to better understand molecular function or biological process difference between circulating miRNA profiles in patients with QSB and QDB syndromes, we compared the gene ontology of miRNAs target genes. Venn diagram displayed the overlapping and nonoverlapping functional annotations in QSB and QDB groups, which showed that 123 upregulated (Figure 2(a)) and 11 downregulated (Figure 2(b)) differentially functional annotations overlapped among the QSB and QDB groups. According to the GO analysis, the obvious upregulated and downregulated functional annotations of the differentially expressed miRNAs target genes in the QSB group (Figure 2(c)) were 


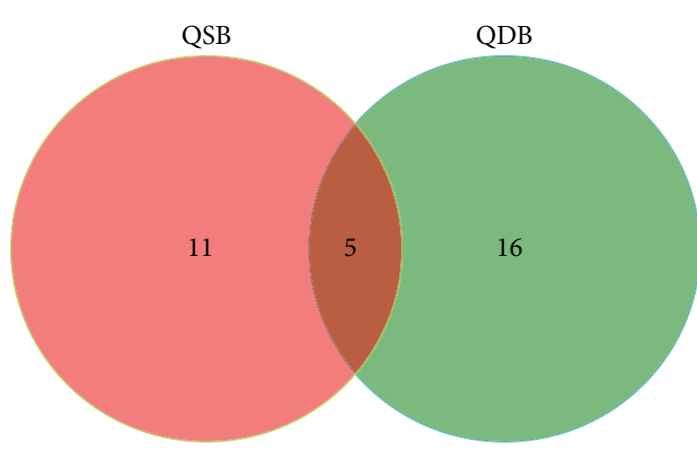

Upregulated

(a)

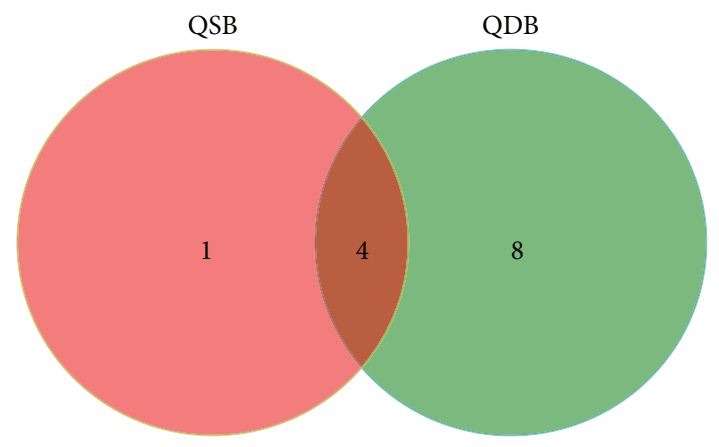

Downregulated

(b)

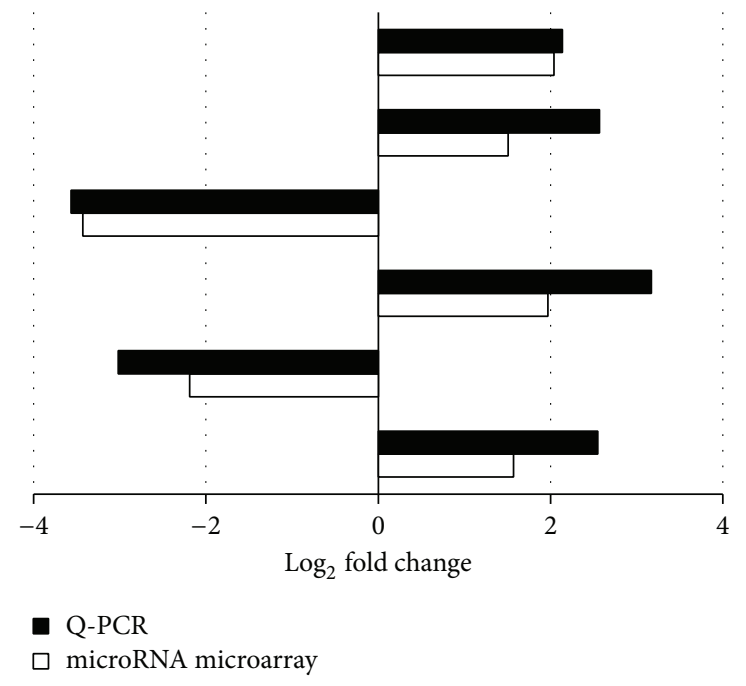

(c)

FIGURE 1: (a) Section of upregulated differentially miRNAs overlapping and nonoverlapping in QSB and QDB groups. (b) Section of downregulated differentially miRNAs overlapping and nonoverlapping in QSB and QDB groups. (c) Real time qRT-PCR validation of the microarray results: microarray results compared to qRT-PCR results relative to the OC. The obvious upregulated or downregulated miRNAs in QSB and QDB groups from microarray data (FDR $P<0.05$; fold change $>1.5$ ) were randomly selected. Expression changes are depicted as $\log _{2}$ fold change ( $x$-axis). miRNAs symbols are shown on the other side of the column.

related to regulation of cellular component organization, regulation of glucose metabolic process, cell-cell signaling, and so forth and protein import into nucleus, nuclear import, nucleocytoplasmic transport, and so forth, respectively. Based on the function of differentially expressed miRNAs target genes in the QDB group (Figure 2(d)), the obvious upregulated and downregulated functional annotations were mainly related to protein kinase cascade, regulation of small GTPase mediated signal transduction, phosphate metabolic process, and so forth and vesicle-mediated transport, negative regulation of macromolecule metabolic process, enzyme linked receptor protein signaling pathway, and so forth, respectively.

3.5. Pathway Analysis of miRNAs Target Genes. Based on the KEGG database, 4 upregulated (Figure 3(a)) and 3 downregulated (Figure 3(b)) overlapping pathways were identified in QSB group and QDB group including pathways in cancer, TGF-beta signaling pathway, and calcium signaling pathway. Moreover, 6 pathways were remarkably upregulated in the QSB group, and 8 and 25 pathways were significantly downregulated in QDB group. Table 2 demonstrated that cell adhesion molecules (CAMs) played the crucial role in the pathological process of Qi-stagnation, with a significant and unique upregulation besides the rest cancer related pathways. Table 3 displayed that Qi-deficiency was associated with endocytosis and mTOR signaling pathway which is involved in the process of autophagy.

3.6. MicroRNA-Gene-Net Analysis. Gene regulatory networks composed of miRNAs and their target mRNAs are expected to be important for the biological function of the specific syndromes. Therefore, we carried out pathway analysis for miRNAs that were differentially expressed between QSB and QDB and that belonged to miRNA clusters or families. The network diagram of QSB group showed the core miRNAs and their target genes including let-7c, miR-4487, miR-619, miR-8075, miR-6735, and miR-32-5p (Figure 3(c)). 


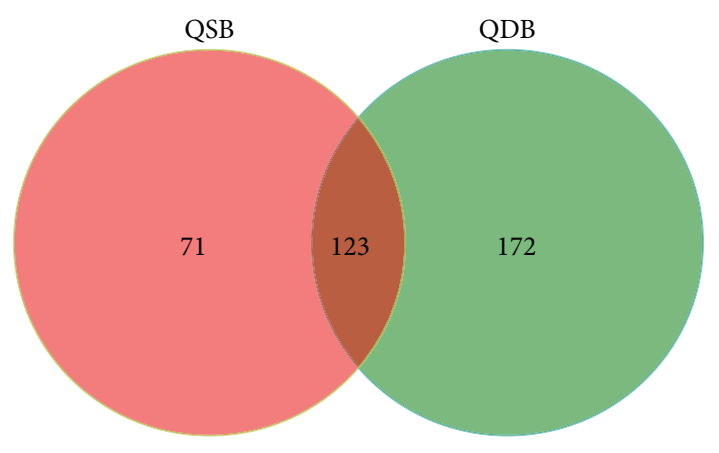

Upregulated

(a)

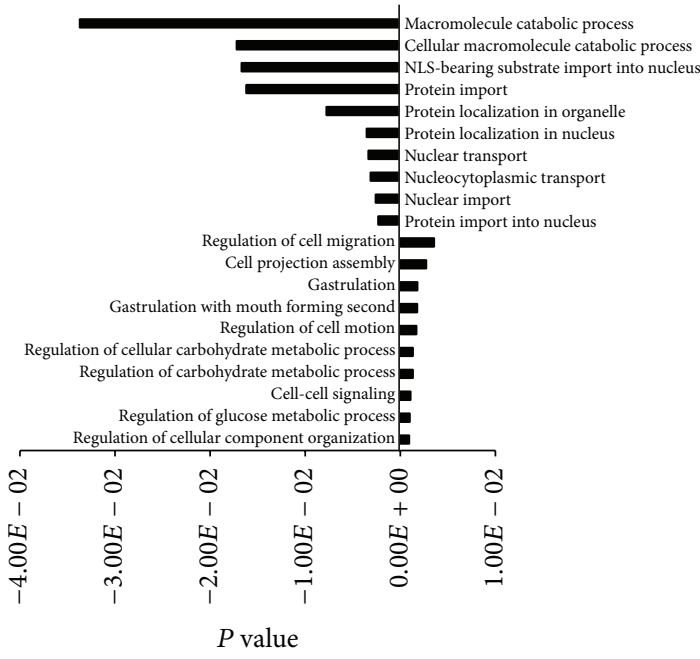

(c)

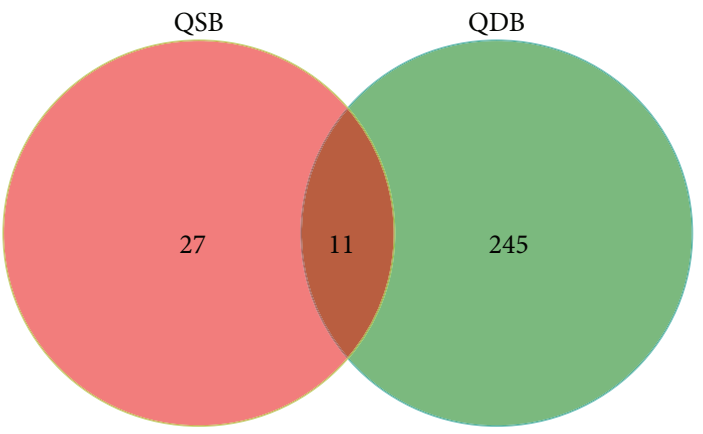

Downregulated

(b)

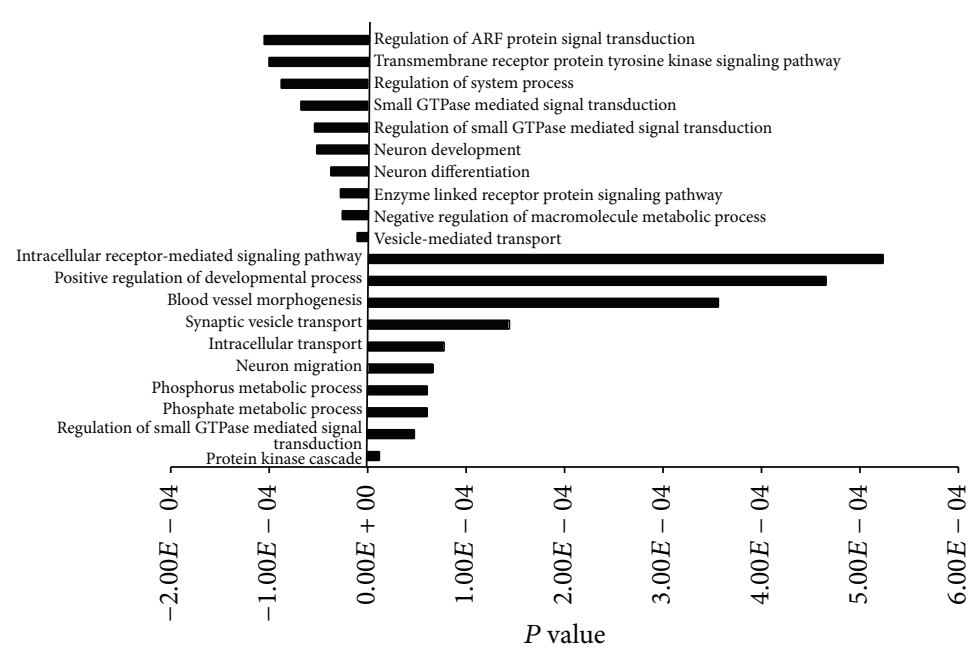

(d)

FIGURE 2: (a) Section of overlapping and nonoverlapping upregulated functional annotations from the differentially expressed miRNAs target genes. (b) Section of overlapping and nonoverlapping downregulated functional annotations from the differentially expressed miRNAs target genes. (c) Bar graphs showed the most obvious upregulated and downregulated functional annotations of QSB group. (d) Bar graphs showed the most obvious upregulated and downregulated functional annotations of QDB group. Expression changes are depicted as $P$ value ( $x$-axis). GO symbols are shown on the other side of the column.

As for QDB group, miR-17-5p, miR-130a, miR-320a, miR$320 \mathrm{~b}$, and miR-320c displayed the important role in the network (Figure 3(d)).

\section{Discussion}

In the long history of traditional clinical practice in China and other Eastern countries, TCM practitioners have typically classified patients of the same disease into subgroups as different syndromes from a holistic perspective on patients' overall status [10]. Qi-blood theory, a complicated and intricate theory, is one of the basic theories of TCM. Qi is used to describe the refined nutritious substances constituting the human body and maintaining life activities. The concept of blood in TCM is also used to describe body's functions and is always regarded the same as the blood in western medicine in most conditions, with a clearer definition than Qi [11]. Qi-blood theory is widely used to in the diagnosis and treatment for the chronic diseases such as CHD [12].
However, Qi-stagnation (QSB) and Qi-deficiency (QDB) symptoms in $\mathrm{CHD}$ patients are subjective and difficult to evaluate objectively. We hypothesized that microRNAs from QSB and QDB appearances may reflect characteristics of the syndromes, which is associated with the status of the Qiblood.

According to the analysis of the differential miRNAs profiles, significantly upregulated or downregulated miRNAs are overlapped in the QSB and QDB groups, such as miR451a, miR-3152, and miR-4487. This indicates that the parts of mechanism for QSB and QDB groups were similar and may be the basis of QSB and QDB syndrome. Based on the upregulated or downregulated value in QSB, the most significant upregulated miRNA is miR-7641 which can significantly suppress CXCL1, a member of the CXC chemokine family that promotes neovascularization by binding G-protein coupled receptors and is related to endothelial cells biogenesis such as angiogenesis [13]. Consequently, the upregulated miR-7641 in QSB patients suggests an abnormal endothelial 


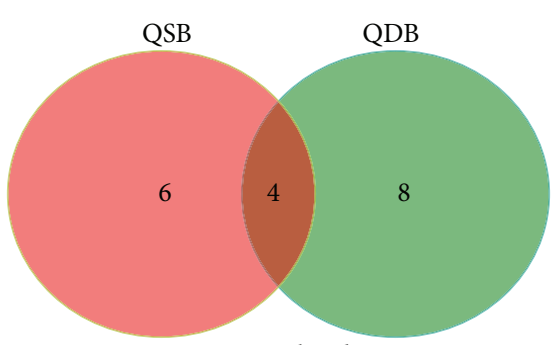

Upregulated

(a)

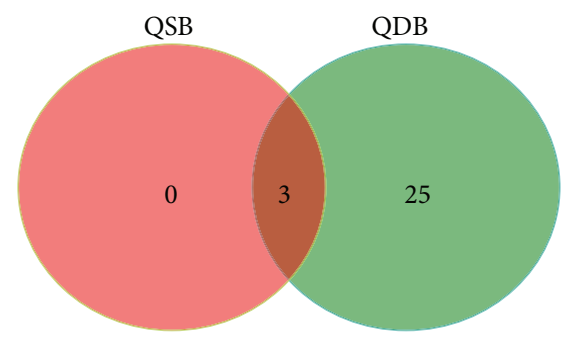

Downregulated

(b)

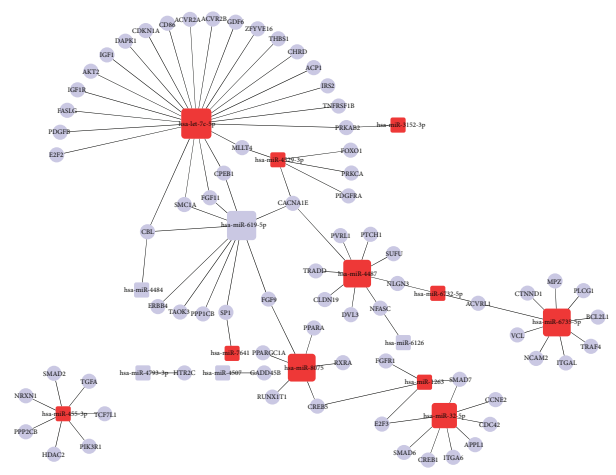

(c)

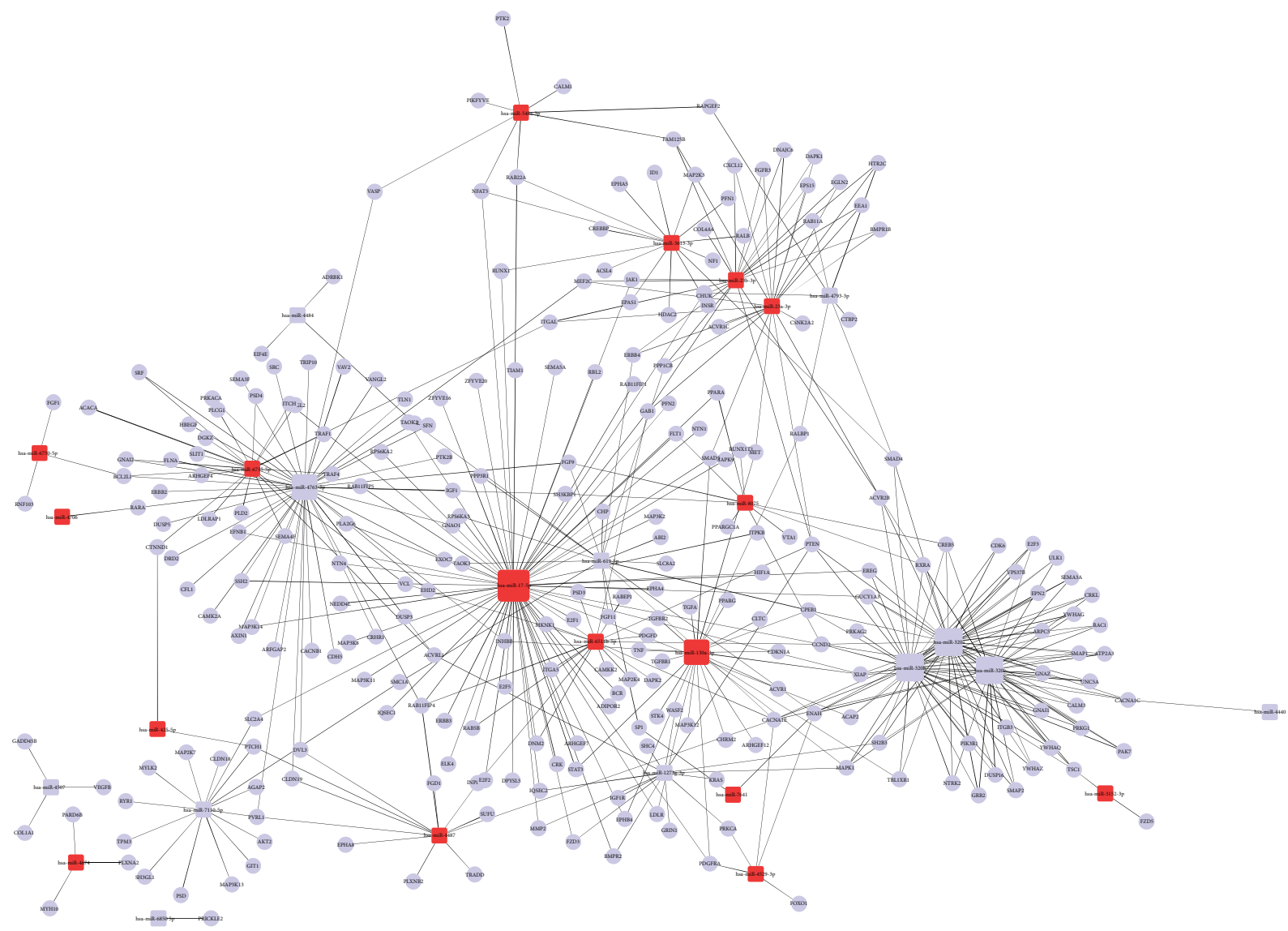

(d)

FIGURE 3: (a) Venn diagram showed overlapping and nonoverlapping upregulated pathways in QSB and QDB groups. (b) Venn diagram showed overlapping and nonoverlapping downregulated pathways in QSB and QDB groups. (c) The microRNA-gene-network diagram of QSB. (d) The microRNA-gene-network diagram of QDB. Red squares represent upregulated miRNAs and the blue squares represent downregulated miRNAs. Blue rounds represent the target genes of their connected miRNAs. Black lines represent the regulatory relation between miRNAs and their target genes. 
TABLE 2: Upregulated pathways in patients with QSB syndrome.

\begin{tabular}{|c|c|c|c|c|c|}
\hline Path_ID & Path_name & Count & $\%$ & $P$ value & FDR \\
\hline hsa05215 & Prostate cancer & 16 & 1.394943 & $2.63 E-04$ & 0.020708 \\
\hline hsa05214 & Glioma & 12 & 1.046207 & 0.00128 & 0.065618 \\
\hline hsa05218 & Melanoma & 12 & 1.046207 & 0.003441 & 0.128043 \\
\hline hsa04514 & Cell adhesion molecules (CAMs) & 17 & 1.482127 & 0.006066 & 0.175925 \\
\hline hsa05222 & Small cell lung cancer & 11 & 0.959024 & 0.030494 & 0.42138 \\
\hline hsa05223 & Non-small cell lung cancer & 8 & 0.697472 & 0.043989 & 0.510943 \\
\hline
\end{tabular}

TABLE 3: (a) Upregulated pathways in patients with QDB syndrome. (b) Downregulated pathways in patients with QDB syndrome.

(a)

\begin{tabular}{lccccc}
\hline Path_ID & Path_name & Count & $\%$ & $P$ value & FDR \\
\hline hsa04144 & Endocytosis & 41 & 0.245627 & $5.65 E-08$ & $9.66 E-06$ \\
hsa04360 & Axon guidance & 28 & 0.167745 & $1.84 E-05$ & 0.00157 \\
hsa05212 & Pancreatic cancer & 15 & 0.089863 & 0.003962 & 0.126948 \\
hsa04010 & MAPK signaling pathway & 36 & 0.215672 & 0.01223 & 0.259629 \\
hsa05220 & Chronic myeloid leukemia & 14 & 0.083873 & 0.014293 & 0.239301 \\
hsa04070 & Phosphatidylinositol signaling system & 13 & 0.077882 & 0.029505 & 0.400777 \\
hsa04810 & Regulation of actin cytoskeleton & 28 & 0.167745 & 0.041864 & 0.485632 \\
hsa04012 & ErbB signaling pathway & 14 & 0.083873 & 0.043572 & 0.469973 \\
\hline
\end{tabular}

(b)

\begin{tabular}{lccccc}
\hline Path_ID & Path_name & Count & $\%$ & $P$ value & FDR \\
\hline hsa04012 & ErbB signaling pathway & 18 & 1.880878 & $1.15 E-05$ & 0.001671 \\
hsa05214 & Glioma & 14 & 1.462905 & $6.91 E-05$ & 0.00503 \\
hsa04910 & Insulin signaling pathway & 21 & 2.194357 & $1.33 E-04$ & 0.006429 \\
hsa04510 & Focal adhesion & 27 & 2.821317 & $1.39 E-04$ & 0.005062 \\
hsa04730 & Long-term depression & 13 & 1.358412 & $6.98 E-04$ & 0.016838 \\
hsa05220 & Chronic myeloid leukemia & 13 & 1.358412 & 0.001496 & 0.030734 \\
hsa04720 & Long-term potentiation & 12 & 1.253918 & 0.002133 & 0.038212 \\
hsa04722 & Neurotrophin signaling pathway & 17 & 1.776385 & 0.002769 & 0.039679 \\
hsa04150 & mTOR signaling pathway & 10 & 1.044932 & 0.003329 & 0.043298 \\
hsa05212 & Pancreatic cancer & 12 & 1.253918 & 0.003394 & 0.04052 \\
\hline
\end{tabular}

cells function which possibly results for the inhibition of angiogenesis. The most significant downregulated miRNA in QSB group is miR-4484 which differentially expressed between macrophages infected with virulent and avirulent mycobacterium tuberculosis [14]. In addition to endothelial cells function, the macrophage dysfunction may be another biological basic of QSB syndrome. According to the $\log _{2}$ fold change value in QDB group, the most obvious downregulated miRNA is miR-320c. It was evidenced that downregulation of miR-320 induced the overexpression of proinflammatory cytokines through the modulation of ERK/NF- $\kappa \mathrm{B}$ pathways [15]. Therefore, in the process of QDB, downregulated miR-320 may contribute to the proinflammatory cytokines generation.

Based on the results of GO analysis, the functions of most differentially regulated genes in QSB were related to the regulation of cellular component organization, protein import into nucleus, glucose metabolic process, and so forth, which indicated that the mechanism for QSB syndrome might relate to the dysfunction of the above biological process. As for the QDB, the functions of most differentially regulated genes were related to protein kinase cascade, regulation of small GTPase mediated signal transduction, vesicle-mediated transport, and so forth, suggesting that QSB syndrome might relate to the dysfunction of the above biological process.

According to the KEGG analysis, 6 pathways were related to the QSB, 5 of which were related to the cancer concerned pathways. The only pathway that did not belong to the cancer concerned pathway was cell adhesion molecules (CAMs). Cell-cell and cell-matrix interactions are mediated through several different families of CAMs including the selectins, the integrins, the cadherins, and the immunoglobulins. CAMs play a very significant and critical role in both heart development and different pathophysiological heart disease [16]. Blood stasis syndrome has a certain degree of high coagulation state. On this pathological basis, cell adhesion molecules were more significant in the QSB than that in QDB which indicated that CAMs play more important role in QSB. We assumed that several members in the CAMs superfamilies and in particular the integrin family will serve as diagnostic strategies with QSB. The number of pathway with obvious change in QDB group is more than QSB group, parts of which are associated with phagocytosis such as endocytosis, 
mTOR signaling pathway which is involved in the process of autophagy. All types of cells in the body use the endocytosis process to communicate with the biological environments. This process is an energy-dependent process through which cells internalize ions and biomolecules [17]. In addition, it is evidenced that the cellular rate of nutrient or ion uptake (e.g., glucose, $\mathrm{Fe}^{3+}$, and $\mathrm{K}^{+}$) or efflux (e.g., $\mathrm{Na}^{+}$) is governed by a complement of membrane transporters and receptors that show dynamic localization at both the plasma membrane and the defined intracellular membrane compartments [18]. Some research showed that endocytosis was upregulated in border zone cardiomyocytes, and inhibition of endocytosis might be an effective approach to prevent export of injury signals from the myocardial infarct size [19]. In considering endocytosis as the most obvious upregulated pathway in QDB, we speculate that endocytosis process consumes excessive the above mentioned nutrients so that the body appears a series of energy deficiency signs, as so-called Qi-deficiency. mTOR signaling pathway is one of regulatory molecules that negatively controls autophagy [20-22]. Consequently, the downregulated mTOR signaling pathway in QDB can activate autophagy which is evidenced to be harmful for myocardial ischemia reperfusion $[23,24]$. The downregulated mTOR signaling pathway in CHD patients with QDB syndrome results in the activation of autophagy which could increase myocardial cell apoptosis. Therefore, the autophagy may be another biological characteristic of QDB syndrome different from QSB syndrome.

The construction of microRNA-gene-network in QSB group demonstrates that let-7c, miR-4487, miR-619, miR8075, miR-6735, and miR-32-5p are located in the center of diagram, suggesting that the abnormal miRNAs may have the most important and extensive regulatory function for Qi-stagnation. The construction of microRNA-genenetwork in QDB displays that miR-17-5p, miR-130a, miR$320 \mathrm{a}, \mathrm{miR}-320 \mathrm{~b}$, and miR-320c are located in the center of diagram, suggesting that the abnormal miRNAs may have the most important and extensive regulatory function for Qideficiency. Further, we found that though miR-4487, miR619 , and miR-8075 were the overlapping miRNAs in both QSB and QDB syndromes, their regulatory function seemed more wide and involved in more regulatory networks in QSB syndromes than that in QDB syndromes. Of note, miR-320 family including miR-320a, miR-320b, and miR320 c regulates QDB syndromes predominantly. MiR-320 has been found to have widespread biological effects as it regulates multiple important molecules. Its potential targets include ET-1, ERK1, VEGF, and FN (http://www.microrna .org/). The biologic actions of miR-320 involve carcinogenesis, development, and ischemia reperfusion injury $[25,26]$. Combined with Qi-deficiency characteristic, we consider that miR-320 family plays a crucial role in various performances with insufficient energy and nutrient.

In this paper, we compared molecular networks of two syndromes of $\mathrm{CDH}$ patients with blood stasis and analyzed their common and different microRNAs mechanisms. We achieve a better understanding of the biological characteristics of syndrome differentiation of CHD which is beneficial to explore different therapies to enhance the effectiveness and pertinence of treatment.

\section{Conflict of Interests}

The authors declare that there is no conflict of interests regarding the publication of this paper.

\section{Authors' Contribution}

Dr. Jincai Hou and Dr. Jun Wang contribute equally to this work and are co-first authors.

\section{Acknowledgments}

This research was supported by the National Basic Research Program of China (973 Program, 2015CB554400); the National Science \& Technology Major Project of China (2012ZX09301002-004-002 and 2012ZX09103201-049); the National Natural Science Foundation of China (81473449 and 81102679); and the Fundamental Research Funds for the Central public welfare research institutes (ZZ070824).

\section{References}

[1] S. S. Lim, T. Vos, A. D. Flaxman et al., "A comparative risk assessment of burden of disease and injury attributable to 67 risk factors and risk factor clusters in 21 regions, 1990-2010: a systematic analysis for the Global Burden of Disease Study 2010," The Lancet, vol. 380, no. 9859, pp. 2224-2260, 2012.

[2] C. J. L. Murray and A. D. Lopez, "Measuring the global burden of disease," The New England Journal of Medicine, vol. 369, no. 5, pp. 448-457, 2013.

[3] A. Lu and K. Chen, "Chinese medicine pattern diagnosis could lead to innovation in medical sciences," Journal of Traditional Chinese Medicine, vol. 17, no. 11, pp. 811-817, 2011.

[4] M. Jiang, C. Lu, C. Zhang et al., "Syndrome differentiation in modern research of traditional Chinese medicine," Journal of Ethnopharmacology, vol. 140, no. 3, pp. 634-642, 2012.

[5] Y. Xing, J. Wang, X. Yang et al., "The effects of Xuefu Zhuyu and Shengmai on the evolution of syndromes and inflammatory markers in patients with unstable angina pectoris after percutaneous coronary intervention: a randomised controlled clinical trial," Evidence-Based Complementary and Alternative Medicine, vol. 2013, Article ID 896467, 9 pages, 2013.

[6] R. C. Friedman, K. K.-H. Farh, C. B. Burge, and D. P. Bartel, "Most mammalian mRNAs are conserved targets of microRNAs," Genome Research, vol. 19, no. 1, pp. 92-105, 2009.

[7] E. M. Small and E. N. Olson, "Pervasive roles of microRNAs in cardiovascular biology," Nature, vol. 469, no. 7330, pp. 336-342, 2011.

[8] S. Fichtlscherer, A. M. Zeiher, and S. Dimmeler, "Circulating MicroRNAs: biomarkers or mediators of cardiovascular diseases?" Arteriosclerosis, Thrombosis, and Vascular Biology, vol. 31, no. 11, pp. 2383-2390, 2011.

[9] W. Q. Li, C. Chen, M. D. Xu et al., "The rno-miR-34 family is upregulated and targets ACSL1 in dimethylnitrosamineinduced hepatic fibrosis in rats," The FEBS Journal, vol. 278, no. 9, pp. 1522-1532, 2011. 
[10] B. Jiang, X. Liang, Y. Chen et al., "Integrating next-generation sequencing and traditional tongue diagnosis to determine tongue coating microbiome," Scientific Reports, vol. 2, p. 936, 2012.

[11] W. Yao, H. Yang, and G. Ding, "Mechanisms of Qi-blood circulation and Qi deficiency syndrome in view of blood and interstitial fluid circulation," Journal of Traditional Chinese Medicine, vol. 33, no. 4, pp. 538-544, 2013.

[12] M. Jiang, C. Zhang, H. Cao, K. Chan, and A. Lu, “The role of Chinese medicine in the treatment of chronic diseases in China," Planta Medica, vol. 77, no. 9, pp. 873-881, 2011.

[13] J. K. Yoo, H. Y. Jung, C.-H. Kim, W. S. Son, and J. K. Kim, "MiR7641 modulates the expression of CXCL1 during endothelial differentiation derived from human embryonic stem cells," Archives of Pharmacal Research, vol. 36, no. 3, pp. 353-358, 2013.

[14] K. Das, S. Saikolappan, and S. Dhandayuthapani, "Differential expression of miRNAs by macrophages infected with virulent and avirulent Mycobacterium tuberculosis," Tuberculosis, vol. 93, pp. S47-S50, 2013.

[15] Z. Cheng, S. Qiu, L. Jiang et al., "MiR-320a is downregulated in patients with Myasthenia gravis and modulates inflammatory cytokines production by targeting mitogen-activated protein kinase 1," Journal of Clinical Immunology, vol. 33, no. 3, pp. 567576, 2013.

[16] S. A. Mousa, "Cell adhesion molecules: potential therapeutic \& diagnostic implications," Molecular Biotechnology, vol. 38, no. 1, pp. 33-40, 2008.

[17] N. Oh and J.-H. Park, "Endocytosis and exocytosis of nanoparticles in mammalian cells," International Journal of Nanomedicine, vol. 9, no. 1, pp. 51-63, 2014.

[18] C. N. Antonescu, T. E. McGraw, and A. Klip, "Reciprocal regulation of endocytosis and metabolism," Cold Spring Harbor Perspectives in Biology, vol. 6, no. 7, Article ID a016964, 2014.

[19] M. Khaidakov, F. Mercanti, X. Wang et al., "Prevention of export of anoxia/ reoxygenation injury from ischemic to nonischemic cardiomyocytes via inhibition of endocytosis," The American Journal of Physiology-Heart and Circulatory Physiology, vol. 306, no. 12, pp. H1700-H1707, 2014.

[20] A. J. Meijer and P. Codogno, "Signalling and autophagy regulation in health, aging and disease," Molecular Aspects of Medicine, vol. 27, no. 5-6, pp. 411-425, 2006.

[21] R. Kang, H. J. Zeh, M. T. Lotze, and D. Tang, "The Beclin 1 network regulates autophagy and apoptosis," Cell Death \& Differentiation, vol. 18, no. 4, pp. 571-580, 2011.

[22] L. Yu, C. K. McPhee, L. Zheng et al., “Termination of autophagy and reformation of lysosomes regulated by Mtor," Nature, vol. 465, no. 7300, pp. 942-946, 2010.

[23] T. Aki, K. Yamaguchi, T. Fujimiya, and Y. Mizukami, "Phosphoinositide 3-kinase accelerates autophagic cell death during glucose deprivation in the rat cardiomyocyte-derived cell line H9c2," Oncogene, vol. 22, no. 52, pp. 8529-8535, 2003.

[24] L. Valentim, K. M. Laurence, P. A. Townsend et al., "Urocortin inhibits Beclinl-mediated autophagic cell death in cardiac myocytes exposed to ischaemia/reperfusion injury," Journal of Molecular and Cellular Cardiology, vol. 40, no. 6, pp. 846-852, 2006.

[25] X.-P. Ren, J. Wu, X. Wang et al., "MicroRNA-320 is involved in the regulation of cardiac ischemia/reperfusion injury by targeting heat-shock protein 20," Circulation, vol. 119, no. 17, pp. 2357-2366, 2009.
[26] B. Feng and S. Chakrabarti, "MiR-320 regulates glucoseinduced gene expression in diabetes," ISRN Endocrinology, vol. 2012, Article ID 549875, 6 pages, 2012. 


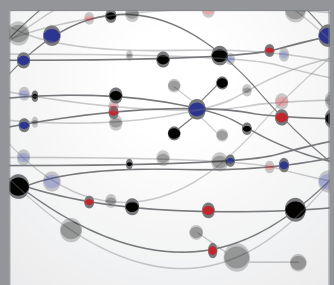

The Scientific World Journal
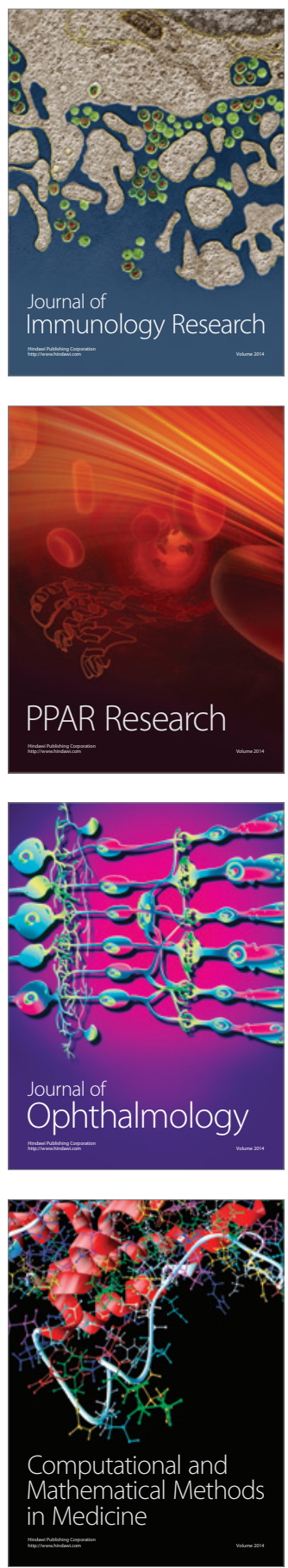

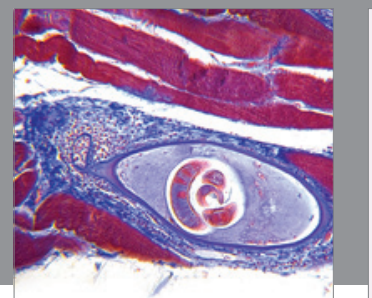

Gastroenterology

Research and Practice
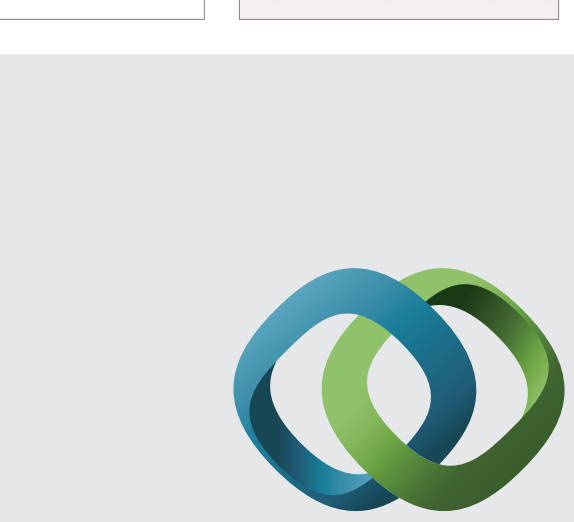

\section{Hindawi}

Submit your manuscripts at

http://www.hindawi.com
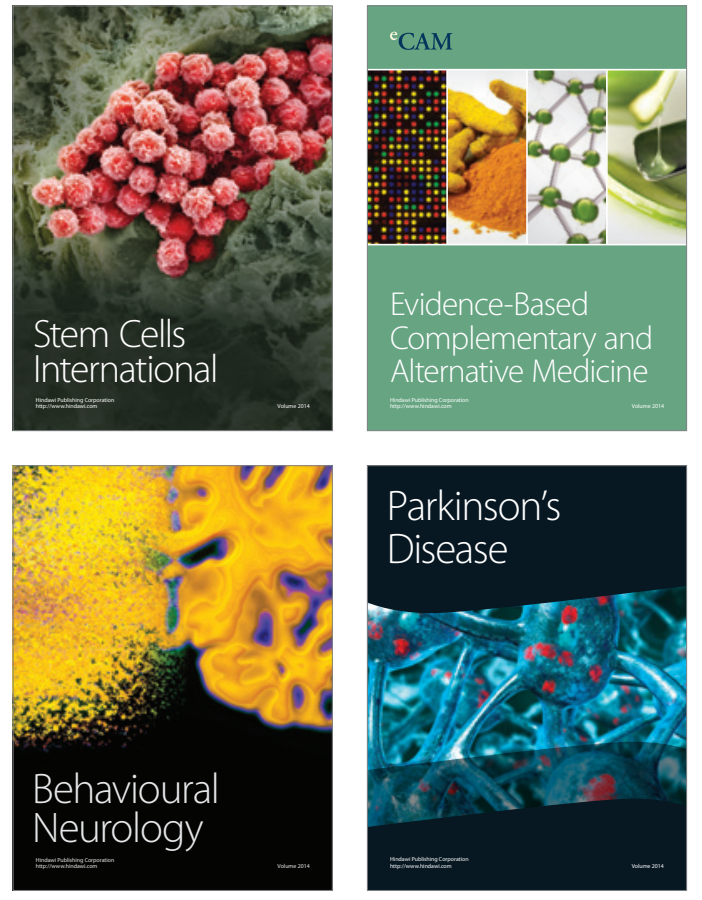
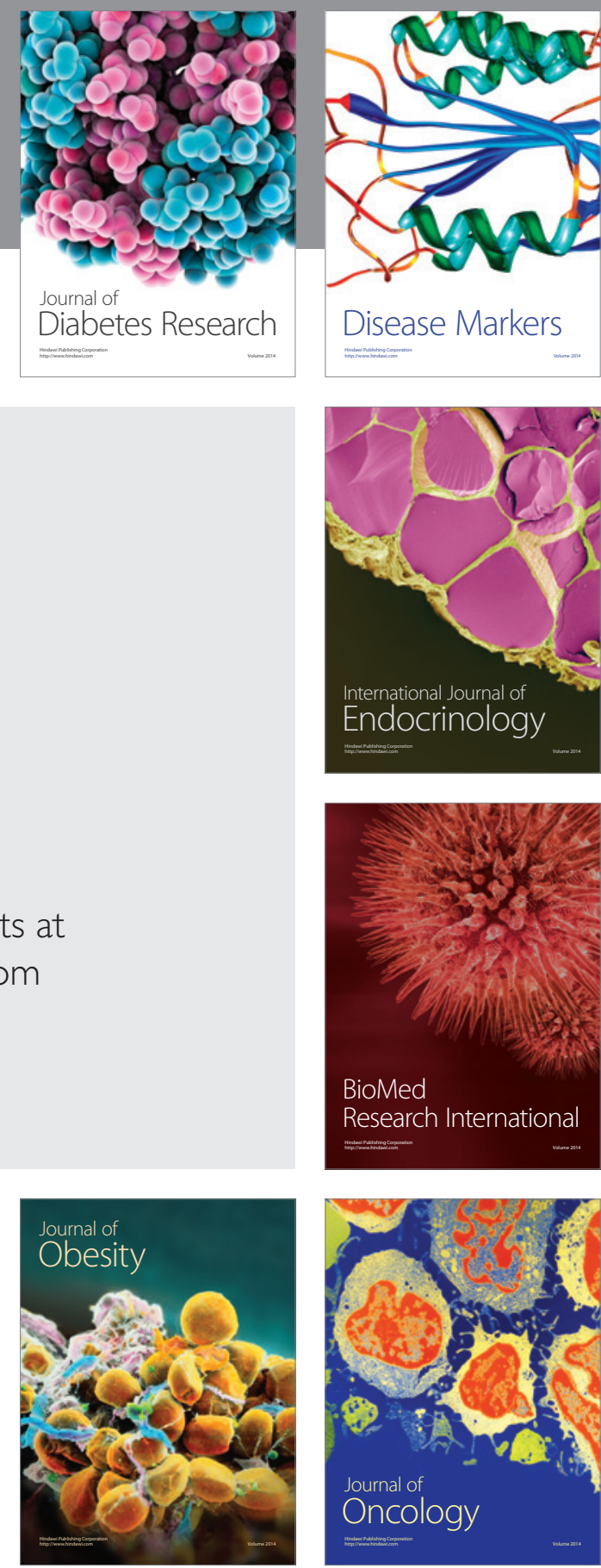

Disease Markers
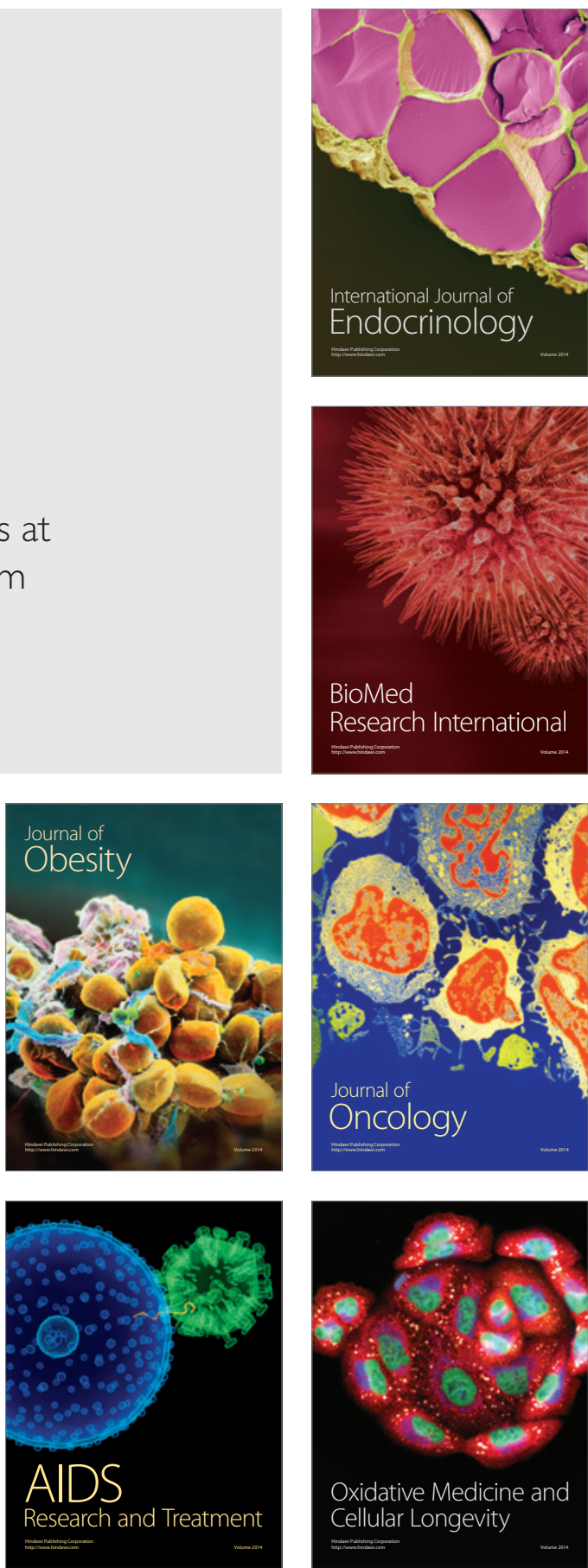